\title{
ON PÓLYA-SZEGÖ AND CHEBYSHEV TYPES INEQUALITIES INVOLVING THE RIEMANN-LIOUVILLE FRACTIONAL INTEGRAL OPERATORS
}

\author{
Sotiris K. Ntouyas, PraveEn Agarwal And Jessada TARiboon
}

\begin{abstract}
In this paper, we investigate some new Pólya-Szegö type integral inequalities involving the Riemann-Liouville fractional integral operator, and use them to prove some fractional integral inequalities of Chebyshev type, concerning the integral of the product of two functions and the product of two integrals. Certain special cases are also considered. Finally, examples for constructing the bounding functions are also given.
\end{abstract}

Mathematics subject classification (2010): 26D10, 26A33, 26D15.

Keywords and phrases: Integral inequalities, Chebyshev functional, Riemann-Liouville fractional integral operator, Pólya-Szegö type inequalities.

\section{REFERENCES}

[1] A. Anber And Z. Dahmani, New integral results using Pólya-Szegö inequality, Acta Comment. Univ. Tartu. Math. 17 (2) (2013), 171-178.

[2] S. Belarbi And Z. Dahmani, On some new fractional integral inequalities, J. Inequal. Pure Appl. Math. 10 (3) (2009), Art. 86, 5 pp. (electronic).

[3] P. L. Chebyshev, Sur les expressions approximatives des integrales definies par les autres prises entre les mêmes limites, Proc. Math. Soc. Charkov 2 (1882), 93-98.

[4] P. CERone AND S. S. DRAGOMir, A refinement of the Grüss inequality and applications, Tamkang J. Math. 38 (2007), 37-49.

[5] Z. Dahmani, O. Mechouar and S. Brahami, Certain inequalities related to the Chebyshev's functional involving a type Riemann-Liouville operator, Bull. Math. Anal. Appl. 3 (4) (2011), 38-44.

[6] S. S. Dragomir, Some integral inequalities of Grüss type, Indian J. Pure Appl. Math. 31 (4) (2000), 397-415.

[7] S. S. Dragomir And N. T. Diamond, Integral inequalities of Grüss type via Pólya-Szegö and Shisha-Mond results, East Asian Math. J. 19 (1) (2003), 27-39.

[8] G. GRÜSs, Über das maximum des absoluten Betrages von $\frac{1}{b-a} \int_{a}^{b} f(x) g(x) d x-\frac{1}{(b-a)^{2}} \int_{a}^{b} f(x) d x$ $\int_{a}^{b} g(x) d x$, Math. Z. 39 (1935), 215-226.

[9] S. L. Kalla AND A. RAO, On Grüss type inequality for hypergeometric fractional integrals, Matematiche (Catania) 66 (1) (2011), 57-64.

[10] A. A. Kilbas, H. M. Srivastava, J. J. Trujillo, Theory and Applications of Fractional Differential Equations, North-Holland Mathematics Studies, 204. Elsevier Science B. V., Amsterdam, 2006.

[11] D. S. Mitrinović, J. E. PeČARIĆ And A. M. Fink, Classical and New Inequalities in Analysis, Mathematics and its Applications, (East European Series) 61, Kluwer Academic Publishers Group, Dordrecht, 1993.

[12] H. ÖĞÜNMEZ AND U. M. ÖZKAN, Fractional quantum integral inequalities, J. Inequal. Appl. 2011, Article ID 787939, 7 pp.

[13] B. G. PachPatte, On multidimensional Grüss type integral inequalities, J. Inequal. Pure Appl. Math. 3 (2) (2002), Article 27. 
[14] G. Pólya And G. Szegö, Aufgaben und Lehrsatze aus der Analysis, Band 1, Die Grundlehren der mathmatischen Wissenschaften 19, J. Springer, Berlin, 1925.

[15] W. SudSUTAD, S. K. NTOUYAS AND J. TARIBOON, Fractional integral inequalities via Hadamard's fractional integral, Abst. Appl. Anal. ID 563096 (2014), 1-11.

[16] G. WANG, P. AgARWAL AND M. CHAND, Certain Grüss type inequalities involving the generalized fractional integral operator, J. Inequal. Appl. (2014) 2014:147. 